Elect. Comm. in Probab. 6 (2001) 31-38

\title{
BALLISTIC DEPOSITION ON A PLANAR STRIP
}

\author{
RAMI ATAR \\ Haifa, Israel \\ email: atar@ee.technion.ac.il \\ SIVA ATHREYA ${ }^{1}$ \\ Department of Mathematics \\ University of British Columbia \\ Vancouver, Canada \\ email: athreya@math.ubc.ca
}

Technion - Israel Institute of Technology,

MIN KANG

Northwestern University,

Illinois, U.S.A

email: mkang@math.nwu.edu

submitted October 10,2000 Final version accepted February 14, 2001

AMS 1991 Subject classification: 60K35

Ballistic, Deposition, Diffusion Limited Aggregation

Abstract

We consider ballistic diffusion limited aggregation on a finite strip $[0, L-1] \times \mathbb{Z}_{+}$in $\mathbb{Z}^{2}$ for some $L \in \mathbb{Z}_{+}$. We provide numerical bounds on the growth in the height process.

\section{Model Dynamics and main result}

Diffusion limited aggregation (DLA) is a model for crystal growth in $\mathbb{Z}^{2}$ starting from an initial seed placed at the origin. A particle is released from "infinity" and performs a simple random walk until it hits a neighbor of the existing cluster where it attaches itself. Then another particle is released and the procedure repeats itself with the crystal growing at each stage. The problem is very hard to analyze and variants of the model have been studied in [5], [3], [2], [4]. For a survey and discussion see [1].

We study a simplification of this model. We consider the strip of width $L$ with its bottom placed on the x-axis. The particles do not perform random walk but choose one of the $L$ columns and slide down. On their slide downwards they attach themselves to the existing cluster as and when they encounter its neighborhood. This seems to be the simplest variation of the DLA model that preserves the attachment mechanism. It turns out that it is simple

\footnotetext{
${ }^{1}$ RESEARCH SUPPORTED IN PART BY A NSERC GRANT AND THE PACIFIC INSTITUTE FOR MATHEMATICAL SCIENCES.
} 
enough in that it can be described by a Markov process on $\mathbb{Z}^{L}$ and yet involved enough to be interesting. Recently, law of large numbers and central limit theorems have been established [6] for a rich family of models that includes the one studied here.

Our purpose is to provide numerical bounds on growth rate of this model. We also enunciate some connections with other problems by indicating some alternative representations of the model. We proceed to define the model rigorously.

- represents a particle in $S_{6}$

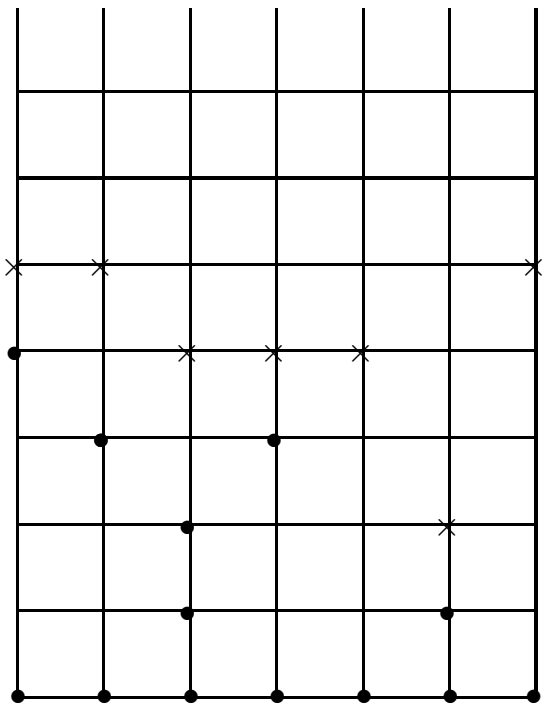

Figure 1: The cluster $S_{6}$ and its boundary.

We consider the $L$-strip $D=[0, L-1] \times \mathbb{Z}_{+}$. We denote the columns by $D_{l}=\{l\} \times \mathbb{Z}_{+}$, $l \in\{0, \ldots, L-1\}$. The symbols $\oplus$ and $\ominus$ stand for addition and subtraction $\bmod L$. For each point $x=(l, m) \in D$ we say that $(l \ominus 1, m+1),(l, m+1)$ and $(l \oplus 1, m+1)$ are the neighbors of $x$, and write $\partial x$ for this set of neighbors. For a cluster $S$ (namely, a finite subset of $D$ ), which intersects all columns $D_{l}, l \in\{0, \ldots, L-1\}$, we define the neighborhood $\partial S$ of $S$ as

$$
\partial S=\left\{(l, m): m \text { is the greatest number } m^{\prime} \text { for which }\left(l, m^{\prime}\right) \in \partial x \text { for some } x \in S\right\} .
$$

Note that, in each column there is exactly one element of $\partial S$.

We begin with an initial cluster of particles $S_{0}=\{0, \ldots, L-1\} \times\{0\}$ and for each $n$ define recursively $S_{n}$ as follows. Let $(\Omega, \mathcal{F}, \mathcal{P})$ be a probability space on which $L$ independent rate 1 Poisson proceses $M_{0}, M_{1}, \ldots M_{L-1}$ are defined. Assume that $\Omega$ is given in a canonical form, i.e $\omega(t)=\left(M_{0}(t, \omega), M_{1}(t, \omega), \ldots M_{L-1}(t, \omega)\right)$. The shift by $s$ operators $\left\{\theta_{s}, s \geq 0\right\}$ act on $\Omega$ in the usual way. Let $\tau_{n}, n=0,1, \ldots$ be the $n$th jump time of any of these processes. Namely, let $\tau_{0}=0$ and for $n \geq 1$ let

$$
\tau_{n}=\inf \left\{t>\tau_{n-1}: M_{l}(t)>M_{l}\left(\tau_{n-1}\right), \text { for some } l\right\} .
$$

Let $l_{n}$ be the index of the Poisson process jumping at $\tau_{n}$, namely the unique $l$ satisfying $M_{l}\left(\tau_{n}\right)=M_{l}\left(\tau_{n-1}\right)+1$. We let $y_{n}$ denote the point where the column $D_{l_{n}}$ intersects $\partial S_{n-1}$, and then let $S_{n}=\left\{y_{n}\right\} \cup S_{n-1}$. For $t \in\left[\tau_{n}, \tau_{n+1}\right)$ we denote $S(t)=S_{n}$. See figure 1 .

We say that $h$ is the height of a cluster $S$ at column $D_{l}$ if $h$ is the greatest number $m$ for which $(l, m) \in S$. We will denote the height of $S(t)$ at column $D_{l}$ by $h_{l}(t)$. The height process 
$\left(h_{l}(t), l \in\{0, \ldots, L-1\}\right), t \geq 0$ is a Markov process which can be described as follows. It changes only at the times $\tau_{n}$ and according to

$$
h_{l}\left(\tau_{n}\right)= \begin{cases}1+h_{l \ominus 1}\left(\tau_{n-1}\right) \vee h_{l}\left(\tau_{n-1}\right) \vee h_{l \oplus 1}\left(\tau_{n-1}\right) & l=l_{n}, \\ h_{l}\left(\tau_{n-1}\right) & l \neq l_{n},\end{cases}
$$

for $n=1,2, \ldots$. The initial condition is $h_{l}(0)=0, l=0, \ldots, L-1$. We also let the maximal height at time $t$ be defined as

$$
H(t)=\max _{0 \leq l \leq L-1} h_{l}(t)
$$

Define for $0 \leq s \leq t, H(s, t)=H(t-s) \circ \theta_{s}$. In words, $H(s, t)$ is the maximal height at time $t-s$, obtained when the model is driven by the processes $\tilde{M}_{i}(t)=M_{i}(t+s), i=0, \ldots, L-1$, $t \geq 0$, rather than the processes $M_{i}(t), i=0, \ldots, L-1, t \geq 0$. It is easy to see that $H(0, s+t) \leq H(0, s)+H(s, t)$ and that $E H(0,1)<\infty$. By Kingman's Sub-additive Ergodic Theorem [7] it follows that a.s.,

$$
\lim _{t \rightarrow \infty} t^{-1} H(t)=\lim _{t \rightarrow \infty} t^{-1} E H(t)=\inf _{t>0} t^{-1} E H(t)=: C_{L} .
$$

One can express the constant $C_{L}$ as a function of the invariant measure of the process $\left\{h_{j \oplus 1}-\right.$ $\left.h_{j}, 0 \leq j \leq L-1\right\}$. However, this measure is not known in an explicit form. We are able to provide bounds on $C_{L}$.

Theorem 1.1 For all $L \geq 4,3.21<C_{L}<5.35$.

One can dominate the height by that of a model that possesses the same transition law as the above model, except that at each time the maximal height hits a multiple of $L$, say $k L$, the heights at all sites $j=0, \ldots, L-1$ are reset to $k L$. This model gives us the upper bound (in Section 2.1). The lower bound is obtained by analyzing a "slower" model, one for which the growth rate is smaller. The simplest example of a slower model is one in which only events (i.e., particle attachments) that immediately increase the maximal height $H$ are accepted, and all other events are ignored. This model easily yields a lower bound of 3 on $C_{L}$. We construct a slightly more complicated model than the one just described. We discuss it in detail in Section 2.2.

Finally in Section 3, we conclude the paper with remarks detailing interesting connections of this model to some other problems.

\section{Proof of Theorem 1.1}

\subsection{An upper bound}

Let $m \in \mathbb{Z}_{+}$be fixed. We define a sequence of stopping times as follows. Let $T_{0}=0$ and for $n=0,1, \ldots$ let $T_{n+1}=\inf \left\{t>T_{n}: H\left(T_{n}, t\right)=m\right\}$. Then $T_{1}$ is simply the first time the maximal height $H(\cdot)$ is equal to $m$, and $T_{n+1}-T_{n}$ is the first time the height $H\left(T_{n}, \cdot\right)$ is equal to $m$. By subadditivity we have that $H\left(T_{n}\right) \leq n m$. Taking limit on a subsequence in $(3)$ shows that a.s.,

$$
C_{L} \leq \lim _{n \rightarrow \infty} \frac{n m}{T_{n}}=\frac{m}{E T_{1}}
$$


where the last equality follows from the facts that $T_{n+1}-T_{n}$ are i.i.d., $E T_{1}<\infty$ and the Law of Large Numbers. We will show that a lower bound on $E T_{1}$ of the order of $m$ holds.

For $a>0$ one has

$$
E T_{1} \geq \operatorname{am}\left(1-P\left(T_{1}<a m\right)\right) .
$$

Let $B_{n}$ be the set of all sequences $b_{0}, \ldots, b_{n}$ with values in $\{0, \ldots, L-1\}$ and such that for $i=1, \ldots, n,\left|b_{i} \ominus b_{i-1}\right| \leq 1$. We say that "a sequence $b_{0}, \ldots, b_{n}$ in $B_{n}$ is a backbone at time $t^{\prime \prime}$ if $b_{0}$ is any number in $\{0, \ldots, L-1\}$ and there exists a sequence $0=t_{0} \leq t_{1} \leq \ldots \leq t_{n} \leq t$ for which $h_{b_{k}}\left(t_{k}\right)=1+h_{b_{k-1}}\left(t_{k-1}\right)$. Using the fact that having a backbone in $B_{n}$ at time $t$ is necessary and sufficient for having $H(t) \geq n$, we have

$$
\begin{aligned}
P\left(T_{1}<a m\right) & =P\left(\bigcup_{b \in B_{m}} b \text { is a backbone at time } a m\right) \\
& \leq \sum_{b \in B_{m}} P(b \text { is a backbone at time } a m) .
\end{aligned}
$$

A naive estimate on each term in this sum, not depending on $b$, is referred to in an aside at the end of this proof. We proceed with a more involved argument. Fix a $b \in B_{m}$. Let $\beta=\beta(b) \subset\{1, \ldots, m\}$ be the set of $i$ for which $b_{i}=b_{i-1}$. Similarly let $\beta^{+}\left[\right.$resp., $\left.\beta^{-}\right]$be the set of $i$ for which $b_{i}=b_{i-1} \oplus 1\left[b_{i}=b_{i-1} \ominus 1\right]$. Note that $\beta, \beta^{+}, \beta^{-}$form a partition of $\{1, \ldots, m\}$. Define $\sigma_{0}=\inf \left\{t>0: M_{b_{0}}(t)>0\right\}$ and for $k \geq 1 \sigma_{k}=\inf \left\{t>\sigma_{k-1}: M_{b_{k}}(t)>M_{b_{k}}\left(\sigma_{k-1}\right)\right\}$. Let $\Delta \sigma_{k}=\sigma_{k}-\sigma_{k-1}, \gamma=\sum_{k \in \beta} \Delta \sigma_{k}$ and $\delta=\sum_{k \in \beta^{+} \cup \beta^{-}} \Delta \sigma_{k}=\sigma_{m}-\gamma$. Define the events

$$
N_{i}^{k}=\left\{M_{i}\left(\sigma_{k}\right)=M_{i}\left(\sigma_{k-1}\right)\right\}
$$

For $b$ to be a backbone at time $a m$, it is necessary that the following hold: $\sigma_{m}<a m$; occurrence of the events $N_{b_{k} \ominus 1}^{k}$ and $N_{b_{k} \oplus 1}^{k}$ for $k \in \beta$; occurrence of the event $N_{b_{k} \oplus 1}^{k}$ for $k \in \beta^{-}$; occurrence of the event $N_{b_{k} \ominus 1}^{k}$ for $k \in \beta^{+}$. Therefore

$P(b$ is a backbone at time $a m)$

$$
\leq E\left\{P\left[N_{b_{k} \ominus 1}^{k}, N_{b_{k} \oplus 1}^{k}, k \in \beta ; N_{b_{k} \oplus 1}^{k}, k \in \beta^{-} ; N_{b_{k} \ominus 1}^{k}, k \in \beta^{+} \mid \sigma_{0}, \ldots, \sigma_{m}\right] 1_{\left(\sigma_{m}<a m\right)}\right\}(8)
$$

Conditioned on $\sigma_{0}, \ldots, \sigma_{m}$, the events $N_{i}^{k}, i \neq b_{k}$ are independent. It follows that (8) is equal to

$$
\begin{aligned}
E\left[\prod_{k \in \beta}\left(e^{-\Delta \sigma_{k}}\right)^{2} \prod_{k \in \beta^{+}}\right. & \left.e^{-\Delta \sigma_{k}} \prod_{k \in \beta^{-}} e^{-\Delta \sigma_{k}} 1_{\left(\sigma_{m}<a m\right)}\right] \\
& =E\left[e^{-\gamma-\sigma_{m}} 1_{\left(\sigma_{m}<a m\right)}\right] \\
& \left.\leq E\left[e^{-\gamma-\sigma_{m}} e^{-\nu \sigma_{m}+\nu a m}\right] \quad \text { for any arbitrary } \nu>0\right) \\
& =E\left[e^{-(2+\nu) \gamma} e^{-(1+\nu) \delta}\right] e^{\nu a m} \\
& =\left(E e^{-(2+\nu) \Delta \sigma}\right)^{|\beta|}\left(E e^{-(1+\nu) \Delta \sigma}\right)^{m-|\beta|} e^{\nu a m} \\
& \quad(\text { where } \Delta \sigma \text { is an } \exp (1) \text { random variable) } \\
& (3+\nu)^{-|\beta|}(2+\nu)^{-(m-|\beta|)} e^{\nu a m} .
\end{aligned}
$$

Next, note that in $B_{m}$ there are $\left(\begin{array}{c}m \\ k\end{array}\right) 2^{m-k}$ sequences $b$ with $|\beta(b)|=k$. Using the bound obtained in (9) for each $b \in B_{m}$ and from the deduction in (6) we conclude that

$$
P\left(T_{1}<a m\right) \leq \sum_{k=0}^{m}\left(\begin{array}{c}
m \\
k
\end{array}\right) 2^{m-k}(3+\nu)^{-k}(2+\nu)^{-(m-k)} e^{\nu a m} .
$$


Let $g(x)=-x \log x-(1-x) \log (1-x)$ for $x \in(0,1)$. Using Sterling's formula it directly follows that $\left(\begin{array}{c}m \\ k\end{array}\right) \leq c m^{c} \exp (m g(k / m))$, where $c$ is a constant independent of $m$ and $k$. Therefore (10) along with this inequality imply that

$$
P\left(T_{1}<a m\right) \leq c m^{c}(m+1) \exp (m G(\nu, a))
$$

where

$$
G(\nu, a)=\sup _{0<x<1}\left[g(x)-(1-x) \log \left(\frac{2+\nu}{2}\right)-x \log (3+\nu)+\nu a\right]
$$

We define

$$
\bar{G}(a)=\inf _{\nu>0} G(\nu, a)
$$

and

$$
a_{0}=\sup \{a>0: \bar{G}(a)<0\} .
$$

To finish the proof of the upper bound we need the following lemma, proved at the end of this section.

Lemma 2.1 Let $a_{0}$ be as defined in (14). Then $1 / a_{0}<5.35$.

Using (11) we can conclude that

$$
\limsup _{m \rightarrow \infty} m^{-1} \log P\left(T_{1}<a m\right) \leq \bar{G}(a)
$$

and hence for $a<a_{0}, P\left(T_{1}<a m\right) \rightarrow 0$ as $m \rightarrow \infty$. Along with (5) we have that for any $\epsilon>0$

$$
E T_{1} \geq \operatorname{am}(1-\epsilon)
$$

for sufficiently large $m$. From (4) we have that $C_{L} \leq \frac{1}{a(1-\epsilon)}$. Sending $\epsilon \downarrow 0$ and $a \uparrow a_{0}$, one obtains

$$
C_{L} \leq \frac{1}{a_{0}}<5.35
$$

Proof of Lemma 2.1: One finds from (12) that

$$
G(\nu, a)=\log \frac{8+3 \nu}{(2+\nu)(3+\nu)}+\nu a .
$$

Observe that

$$
\frac{\partial G(\nu, a)}{\partial \nu}=-A(\nu)+a, \text { where } A(\nu)=\frac{3 \nu^{2}+16 \nu+22}{(8+3 \nu)(2+\nu)(3+\nu)} .
$$

One can check that $\frac{d A}{d \nu}<0$ for $\quad \nu>0$. Hence for any $a \in(0,22 / 48)$ there is exactly one $\nu_{0}=\nu_{0}(a)>0$ such that $A\left(\nu_{0}\right)=a$. Let $\bar{G}$ be as defined in (13). We have

$$
\frac{d}{d a} \bar{G}(a)=\frac{\partial}{\partial \nu} G\left(\nu_{0}(a), a\right) \frac{\partial \nu_{0}}{\partial a}+\frac{\partial}{\partial a} G\left(\nu_{0}(a), a\right)=\nu_{0}(a)>0, \quad 0<a<\frac{22}{48} .
$$

As a result, the equation $\bar{G}(a)=0$ has at most one solution $a_{0}$. For $\nu=2.477$ (by substitution) we get $\bar{G}(A(\nu))=G(\nu, A(\nu))>0$ and $\bar{G}(A(\nu))<0$ for $\nu=2.478$. It follows that $a_{0}>$ $A(2.478)=0.1870$ and $1 / a_{0}<5.35$. 
Aside: One can obtain an upper bound, albeit less sharp, for $C_{L}$ via a simpler but rougher large deviations estimate. Proceed as indicated in the above proof till (6). Then denote by $S_{m}$, the sum of $m$ i.i.d. standard exponential random variables $\left(s_{i}\right)$, and for $\lambda>0$ arbitrary, use Chebycheff's inequality to get

$$
\begin{aligned}
P\left(T_{1}<a m\right) & \leq L 3^{m} P\left(S_{m}<a m\right)=L 3^{m} P\left(\sum_{1}^{m}-s_{i}>-a m\right) \leq L 3^{m} e^{a \lambda m}\left(E e^{-\lambda s_{1}}\right)^{m} \\
& =L 3^{m} e^{a \lambda m}\left(\frac{1}{1+\lambda}\right)^{m}=L e^{(a \lambda-\log (1+\lambda)+\log 3) m} .
\end{aligned}
$$

Optimizing over $\lambda$ gives, at $\lambda=(1-a) / a$, the estimate $L e^{(1-a+\log 3 a) m}$. Then proceed as indicated in the proof of Theorem 1.1 after (15) to get $a_{0}^{-1}<7.1$.

\subsection{A lower bound}

We obtain lower bounds by considering models whose height processes are dominated by that of the model of interest. Recall that the clusters are defined via the recursion

$$
S_{n}=\left\{y_{n}\right\} \cup S_{n-1},
$$

where $y_{n}$ is where $\partial S_{n-1}$ intersects $D_{l_{n}}$. The idea is to modify the recursive definition and let

$$
\tilde{S}_{n}=\left[\left\{y_{n}\right\} \cup \tilde{S}_{n-1}\right] \cap F\left(l_{n}, S_{n-1}\right),
$$

where $F$ is some function taking values in the set of subsets of $D$. Here $y_{n}$ is where $\partial \tilde{S}_{n-1}$ intersects $D_{l_{n}}$, and if the intersection is empty, then with an abuse of notation, $\left\{y_{n}\right\}$ is regarded as the empty set.

The case $F \equiv D$ is equivalent to (1). Suppose we couple our model of interest with the model (18) so that they are defined on the same probability space and so that $\left\{\tau_{n}\right\}$ and $\left\{l_{n}\right\}$ come from the same set of Poisson processes. Then by induction, the height in (1) is greater than or equal to the height process associated with (18) for all $t \geq 0$ a.s.

The advantage of considering (18) is that one can restrict to a recursion within a set of clusters all of which are vertical shifts of only a finite number of clusters. The simplest example would arise if all clusters are singletons. This corresponds to taking

$$
F\left(l_{n}, \tilde{S}_{n-1}\right)= \begin{cases}\left\{y_{n}\right\} & \partial \tilde{S}_{n-1} \cap D_{l_{n}} \neq \emptyset \\ \tilde{S}_{n-1} & \text { otherwise }\end{cases}
$$

where $y_{n}$ is defined as before. The height in this model grows by 1 each time $D_{l_{n}}$ intersects $\partial \tilde{S}_{n-1}=\partial y_{n-1}$. Therefore, the height grows at rate 3 , and as a result we get that $C_{L} \geq 3$.

Heuristically, the way in which the above model simplifies (1) is by keeping track of one component and ignoring the events that do not immediately contribute to its growth. In the next model we consider, less such events are ignored. Here $\tilde{S}_{n}$ takes values in the set of clusters that are vertical and horizontal shifts of the five 'basic clusters' depicted at Figure 2. The precise definition of $F$ for this model is cumbersome, and is therefore skipped. However, the dynamics of the model should be clear from the following example. Suppose $\tilde{S}_{n-1}$ is a shifted version of cluster 3 (in Figure 2), located at columns $D_{l}$ and $D_{l+1}$. Then if $l_{n}=l-1$, $F=D_{l_{n}-1} \cup D_{l_{n}} \cup D_{l_{n}+1}$, which results with a cluster $\tilde{S}_{n}$ that is a shifted version of cluster 4 . 
Similarly, if $l_{n}=l, l+1, l+2$, respectively, then $S_{n}$ will be a shifted version of cluster $1,2,3$, respectively.

One obtains a five state Markov process, which is analyzed as follows. The intensity matrix is

$$
G=\left(\begin{array}{rrrrr}
-3 & 1 & 1 & 1 & 0 \\
1 & -2 & 1 & 0 & 0 \\
1 & 1 & -3 & 1 & 0 \\
1 & 2 & 1 & -5 & 1 \\
1 & 1 & 1 & 2 & -5
\end{array}\right)
$$

The corresponding invariant distribution is

$$
p=\left(\begin{array}{ccccc}
\frac{1}{4} & \frac{17}{46} & \frac{1}{4} & \frac{5}{46} & \frac{1}{46}
\end{array}\right)
$$

As a result, the growth rate of this model is

$$
3[p(1)+p(2)+p(3)+p(5)]+5 p(4)=3.21
$$

and we conclude that $C_{L} \geq 3.21$ for $L \geq 4$.

A more careful analysis based on a larger number of basic clusters results with a bound of $C_{L}>3.25$.

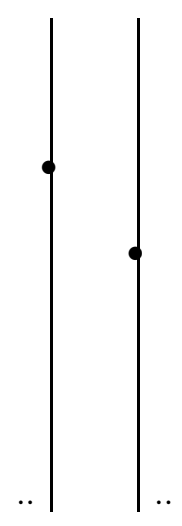

1

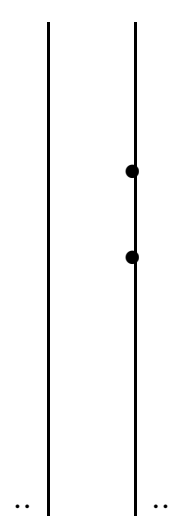

2

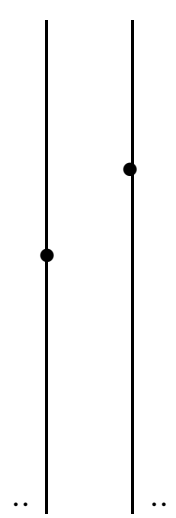

3

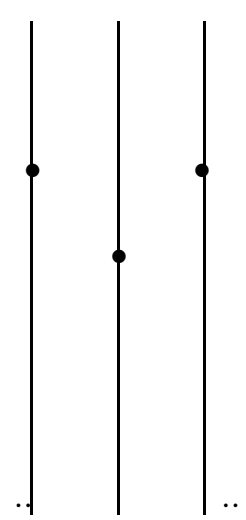

4

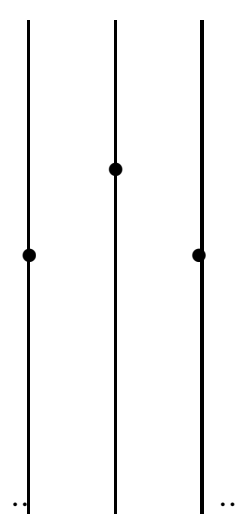

5

Figure 2: A model with five basic clusters

\section{Remarks}

We remark on some alternative ways to represent the model.

(a) Longest increasing subsequence problem.

The asymptotic of the longest length of an increasing subsequence of a random permutation of $\{1, \ldots, n\}$ and that of a sequence of $n$ random drawings from $\{1, \ldots, n\}$ has been the subject

of much research. Although the mathematical structure of the current problem seems rather 
unrelated, we point out that the height in the model discussed here equals the longest length of a subsequence which changes by $-1,0$ or 1 of a sequence of $n$ random drawings from $\{1, \ldots, L\}$.

(b) Products of random matrices.

There is an equivalent formulation of the problem in terms of products of certain random matrices. For $b \in \mathbb{R}$ consider the $L \times L$ matrices $A_{l}, l=1, \ldots, L$ with

$$
A_{l}(i, j)= \begin{cases}1 & i=j \neq l \\ b & i=l, j-l=0 \text { or } \pm 1 \bmod L, \\ 0 & \text { otherwise }\end{cases}
$$

Let $X_{n}$ be a sequence of i.i.d. random matrices where the law of $X_{1}$ is uniform on $\left\{A_{l}, l=\right.$ $1, \ldots, L\}$. Consider $M_{n}=X_{n} X_{n-1} \cdots X_{1}$ and let $e$ denote the column vector of length $L$ with entries 1 . Note that the $i$ th entry $P_{i}(b)$ of the vector $M_{n} e$ is a polynomial in $b$. If one considers a coupling to the model studied in Section 1 , in such a way that $X_{n}=A_{l_{n}}$ for every $n$, then one checks that the height $h_{i}\left(t_{n}\right)$ of the $i$ th column (where $t_{n}$ is the $n$th event) is given by the degree of $P_{i}$. Hence the height $H\left(t_{n}\right)$ is given by the degree of the polynomial $P(b)=e^{\prime} M_{n} e$.

Acknowledgments: The work was done when the three of us were visiting the Fields Institute for Research in Mathematical Sciences in Toronto, Canada. We would like to thank the staff for their hospitality, Jeremy Quastel for introducing us to ballistic deposition, Gregory Lawler and Richard Durrett for helpful discussions.

\section{References}

[1] M. Barlow, Fractals and diffusion-limited aggregation, Bull. Sc. Math, 117:161-169,1993.

[2] M. Barlow, R. Pemantle and E. Perkins, Diffusion-limited aggregation on a tree, Probability Theory and Related Fields, 107:1-60, 1997.

[3] M. Bramson, D. Griffeath, and G. Lawler. Internal diffusion limited aggregation. The Annals of Probability, 20:2117-2140,1992.

[4] D. Eberz, Ph.D. thesis, University of Washington, 1999.

[5] H. Kesten, How long are the arms in DLA.?, J. Phys. A, 20:L29-L33,1987.

[6] M. Penrose and J. Yukich, Limit theory for random sequential packing and deposition, preprint.

[7] A. Shiryaev, Probability, Graduate texts, Springer-Verlag, New York 1995. 